\title{
Unconventional anomalous Hall effect enhanced by a noncoplanar spin texture in the frustrated Kondo lattice Pr2Ir2O7
}

\section{$\operatorname{AUTHOR(S):~}$}

Machida, Y; Nakatsuji, S; Maeno, Y; Tayama, T; Sakakibara, T; Onoda, S

\section{CITATION:}

Machida, Y ... [et al]. Unconventional anomalous Hall effect enhanced by a noncoplanar spin texture in the frustrated Kondo lattice Pr2Ir207. Physical Review Letters 2007, 98(5): 057203.

ISSUE DATE:

2007-02-02

URL:

http://hdl.handle.net/2433/49987

RIGHT:

Copyright 2007 American Physical Society 


\title{
Unconventional Anomalous Hall Effect Enhanced by a Noncoplanar Spin Texture in the Frustrated Kondo Lattice $\operatorname{Pr}_{2} \mathrm{Ir}_{2} \mathrm{O}_{7}$
}

\author{
Y. Machida, ${ }^{1}$ S. Nakatsuji, ${ }^{1,2}$ Y. Maeno, ${ }^{1}$ T. Tayama,${ }^{2}$ T. Sakakibara, ${ }^{2}$ and S. Onoda ${ }^{3}$ \\ ${ }^{1}$ Department of Physics, Kyoto University, Kyoto 606-8502, Japan \\ ${ }^{2}$ Institute for Solid State Physics (ISSP), University of Tokyo, Kashiwa 277-8581, Japan \\ ${ }^{3}$ CREST, Department of Applied Physics, University of Tokyo, Tokyo 113-8656, Japan
}

(Received 13 October 2006; published 30 January 2007)

\begin{abstract}
We have investigated the Hall effect in the geometrically frustrated Kondo lattice $\operatorname{Pr}_{2} \operatorname{Ir}_{2} \mathrm{O}_{7}$. In its spinliquid-like paramagnetic regime, the Hall resistivity $\rho_{x y}$ is found to increase logarithmically on cooling. Moreover, in this low temperature region, the field dependence of the Hall conductivity $\sigma_{x y}$ shows a large enhancement up to $30 \Omega^{-1} \mathrm{~cm}^{-1}$ as well as a nonmonotonic change with the magnetization. Our results are far different from the anomalous Hall effect due to the spin-orbit coupling observed in ordinary magnetic conductors. We discuss the possible spin-chirality effect in the Ir $5 d$ conduction band due to the noncoplanar texture of $\operatorname{Pr}\langle 111\rangle$ Ising-like moments.
\end{abstract}

DOI: 10.1103/PhysRevLett.98.057203

PACS numbers: 75.47. $-\mathrm{m}, 72.15 .-\mathrm{v}, 75.30 . \mathrm{Mb}$

The anomalous Hall effect of magnetic conductors has attracted great interest both in the field of fundamental science and applications. Empirically, the Hall resistivity $\rho_{x y}$ is written as

$$
\rho_{x y}=R_{0} B+4 \pi R_{\mathrm{s}} M,
$$

where $B$ is the magnetic field, and $M$ is the magnetization [1]. The first term is the ordinary Hall resistivity due to the Lorentz force. The second term describes the anomalous Hall effect (AHE) whose origin is theoretically attributed to the magnetization and spin-orbit coupling [2-4]. This relation holds in most magnetic conductors, including ferromagnets, paramagnets, and Kondo lattice systems. Because the AHE can be used to probe $M$, and for its possible application in spintronics, giant effects have been sought for instance in ferromagnetic semiconductors [5,6].

Recently, a new mechanism of the AHE, related to the spin chirality under noncoplanar spin configurations, has been proposed [7-12]. A finite spin chirality, which is proportional to a solid angle subtended by spins, induces a finite Berry phase of conduction electrons. This Berry phase acts as a fictitious magnetic field on the conduction electrons and leads to the AHE [9]. In contrast with the conventional mechanisms, this contribution appears without the spin-orbit coupling. Besides, for the spin-chirality mechanism, a ferromagnet is not necessarily ideal to induce a large Hall effect because the ferromagnetic correlation tends to align conduction electron spins parallel and decreases the chirality.

Up to now, however, the anomalous Hall effect driven by the spin chirality has been experimentally examined mainly in ferromagnets such as manganites $[7,8]$ and pyrochlore molybdates [13-16]. In contrast, a paramagnetic metal with a noncoplanar spin texture is ideal to search for a large Hall effect and to test the validity of the spinchirality mechanism. Such magnets are quite rare, although a number of itinerant systems are known, and thus the recently discovered geometrically frustrated Kondo lattice $\mathrm{Pr}_{2} \mathrm{Ir}_{2} \mathrm{O}_{7}[17,18]$ stands uniquely for such possibility.

In this system, the localized $\operatorname{Pr} 4 f$ moments with $\langle 111\rangle$ Ising anisotropy form a pyrochlore lattice and provide the noncoplanar spin texture $[18,19]$. Ir $5 d$-conduction electrons are only weakly correlated and Pauli paramagnetic. Significantly, because of strong geometrical frustration, $\mathrm{Pr}_{2} \mathrm{Ir}_{2} \mathrm{O}_{7}$ has no magnetic long-range order down to $120 \mathrm{mK}$, despite an antiferromagnetic RKKY interaction of $T^{*}=20 \mathrm{~K}$ between the Pr spins. Instead, the Kondo effect emerges and leads to partial screening of the $4 f$ moments below the Kondo temperature $T_{\mathrm{K}}\left(\sim T^{*}\right)$. Through the renormalization effect, the antiferromagnetic correlation among the resultant underscreened moments become much weaker, and the Weiss temperature decreases by 1 order of magnitude to $\theta_{\mathrm{W}}=1.7 \mathrm{~K}$. On further cooling, the susceptibility exhibits a logarithmically diverging behavior, suggesting that a ferromagnetic coupling eventually develops in this low $T$ regime. Correspondingly, the magnetic part of the specific heat shows a broad peak at $\theta_{\mathrm{W}}$. These indicate that the Pr spins form a correlated liquidlike state below $\theta_{\mathrm{W}}$.

This low $T$ regime is very interesting because the $\operatorname{Pr}$ $\langle 111\rangle$ Ising-like spins are likely to be aligned to yield a finite spin chirality under applied fields, and may induce the fictitious magnetic field on the $5 d$ conduction band through the Kondo coupling [11-14]. This provides a significant and rare test case to find the possible large Hall effect in paramagnetic materials and to check the validity of the spin-chirality mechanism. In this Letter, we report the observation of the divergingly enhanced low $T$ Hall resistivity in the spin-liquid-like regime of the frustrated Kondo lattice $\operatorname{Pr}_{2} \mathrm{Ir}_{2} \mathrm{O}_{7}$. In this regime, $\rho_{x y}$ under a field of $0.3 \mathrm{~T}$ increases logarithmically on cooling and reaches a large value up to $\sim 3 \mu \Omega \mathrm{cm}$, which is comparable with or larger than typical values for ordinary ferro- 
magnets. Furthermore, in the same $T$ region, strong anisotropy and nonmonotonic dependence on $M$ have been observed in $\rho_{x y}$, which sharply contrasts with the empirical relation given by Eq. (1). A possible spin-chirality mechanism is discussed.

Single crystals of $\operatorname{Pr}_{2} \mathrm{Ir}_{2} \mathrm{O}_{7}$ were grown by a flux method [20]. Four-axis x-ray diffraction analysis confirmed that the single crystals have a well-ordered pyrochlore structure with $F d \overline{3} m$ symmetry [20]. Energy dispersive x-ray analysis measurements confirmed the homogeneity and high purity of the crystals. The longitudinal and transverse resistivities ( $\rho_{x x}$ and $\rho_{x y}$ ) were measured by a conventional four-probe method with a current path along the [110] direction. Three different samples from the same batch were used with respect to different field directions. Samples were polished down to a typical size of $0.3 \times$ $0.2 \times 0.05 \mathrm{~mm}^{3}$ and a mass $\sim 0.03 \mathrm{mg}$. We eliminated the longitudinal voltage drop by reversing the field direction. The magnetization $M$ below $2 \mathrm{~K}$ was measured by the Faraday balance method [21].

Figure 1 shows the temperature dependence of $\rho_{x y}(T)$ under a field of $0.3 \mathrm{~T}$ along the [111] direction. From a small value at high $T \mathrm{~s}, \rho_{x y}(T)$ starts increasing substantially below $T^{*}=20 \mathrm{~K}$ and shows an unusual logarithmic increase below $\theta_{\mathrm{W}}=1.7 \mathrm{~K}$. At the lowest temperature $0.4 \mathrm{~K}$, it reaches a large value around $3 \mu \Omega \mathrm{cm}$.

In order to check the expected behavior based on the empirical rule of Eq. (1), we plot in the inset of Fig. 1 the Hall coefficient $R_{H}\left(=\rho_{x y} / B\right)$ vs the susceptibility $4 \pi M / B$ measured under $B=0.3 \mathrm{~T}$ along the [111] direction. A good fit to the experimental data using Eq. (1) is obtained over a decade of temperature between $T^{*}$ and $\theta_{w}$. The obtained value of $R_{s}=0.40 \mathrm{~cm}^{3} / \mathrm{C}$ is about 300 times larger than $R_{0}=-1.51 \times 10^{-3} \mathrm{~cm}^{3} / \mathrm{C}$, as observed in

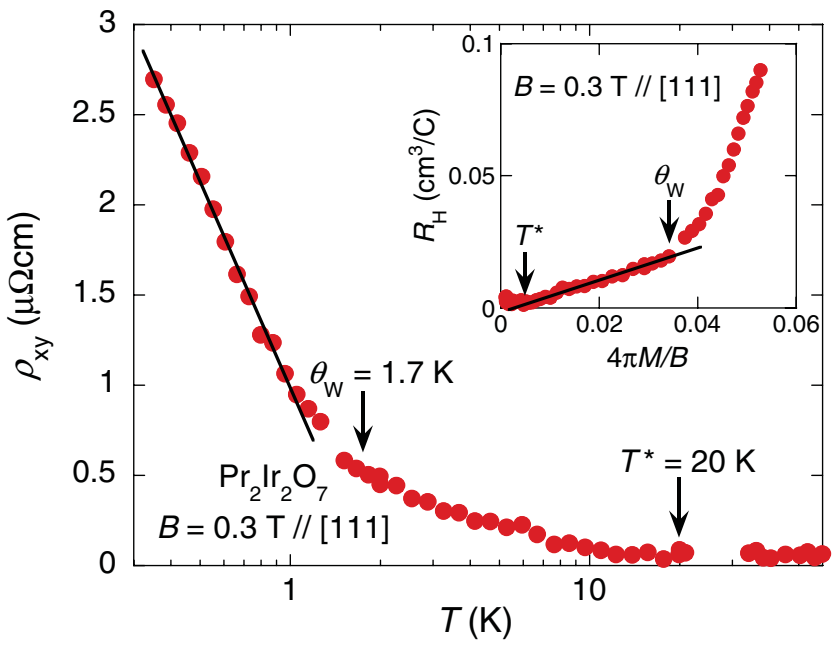

FIG. 1 (color online). Temperature dependence of the Hall resistivity $\rho_{x y}(T)$ under a field of $B=0.3 \mathrm{~T}$ along the [111] direction. Inset: the Hall coefficient $R_{H}$ vs the susceptibility $4 \pi M / B$ under a field of $B=0.3 \mathrm{~T}$ along the [111] direction. ordinary magnetic metals [1]. The single-band relation, $R_{0}=-1 / n e$, yields $n=4.13 \times 10^{21} \mathrm{~cm}^{-3}$, which indicates the low carrier concentration of this system. Using this concentration and the relation $k_{F}=\left(3 \pi^{2} n\right)^{1 / 3}$ for the free electron gas, the RKKY interaction between the nearest neighbor Pr moments is expected to be ferromagnetic. It is likely that through the renormalization of the magnetic correlation, the ferromagnetic nearest neighbor coupling may evolve on cooling and lead to the observed $\ln T$ divergence of the susceptibility below $\theta_{w}$.

In sharp contrast with the ordinary behavior observed in the higher $T$ region, the system below $\theta_{w}$ no longer follows the empirical rules based on the conventional mechanism. First, the temperature dependence of $\rho_{x y}$ shown in the inset of Fig. 1 clearly exhibits a deviation from Eq. (1) below $\theta_{w}$. Figure 2 shows the temperature dependence of the anomalous Hall coefficient $R_{s}(T)$ together with $\rho_{x x}(T)$. Here, because $R_{0} \ll R_{s}, R_{s}$ is estimated to be $\rho_{x y}(T) / 4 \pi M(T)$ at $B=0.3 \mathrm{~T}$. In the low fields $\lesssim 0.3 \mathrm{~T}, \rho_{x y}$ is linearly dependent on $M$ (inset of Fig. 2). Based on the conventional mechanism, $\rho_{x y}$ or $R_{s}$ is known to exhibit a powerlaw dependence on $\rho_{x x}$, i.e., $\rho_{x y}, R_{s} \propto \rho_{x x}^{\alpha}$ with the exponent $\alpha \sim 0.4,1.0$ or 2.0 [22]. Such behavior is indeed observed in $\operatorname{Pr}_{2} \operatorname{Ir}_{2} \mathrm{O}_{7}$ above $\theta_{w}$ as both $R_{s}(T)$ and $\rho_{x x}(T)$ are nearly constant within the experimental error over a decade of temperature between $T^{*}$ and $\theta_{w}$. Below $\theta_{w}$, however, $R_{s}(T)$ no longer obeys such formula but increases nearly logarithmically while $\rho_{x x}(T)$ is constant. This behavior clearly indicates a failure of the empirical relation in this low $T$ regime.

Moreover, the field dependence of the Hall resistivity in the low $T$ regime does not obey the empirical rule of Eq. (1), but shows strong anisotropy and nonmonotonic $M$ dependence. Figs. 3(a) and 3(b) show the field depen-

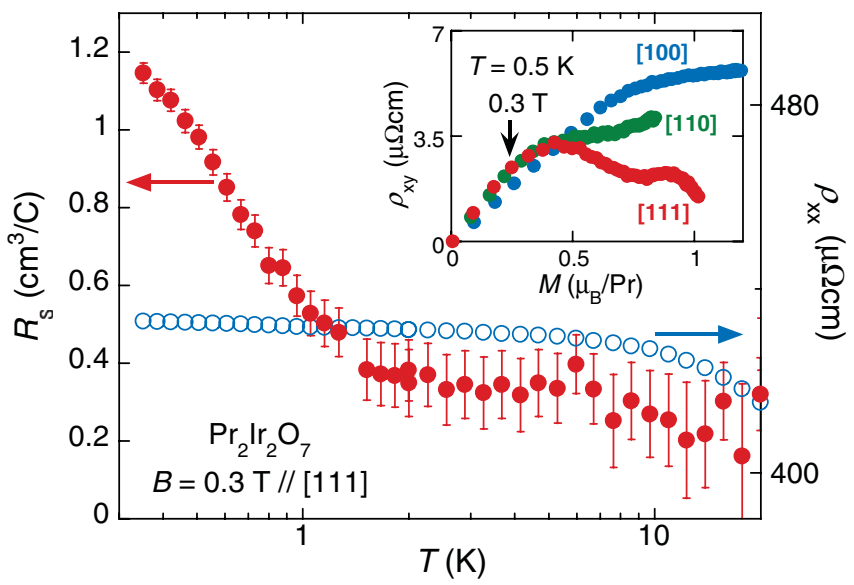

FIG. 2 (color online). Temperature dependence of the anomalous Hall coefficient $R_{s}(T)$ (left) and the longitudinal resistivity $\rho_{x x}(T)$ (right) under a field of $B=0.3 \mathrm{~T}$ along the [111] direction. Inset: $\rho_{x y}$ vs $M$ plot at $T=0.5 \mathrm{~K}$ for different field directions. 

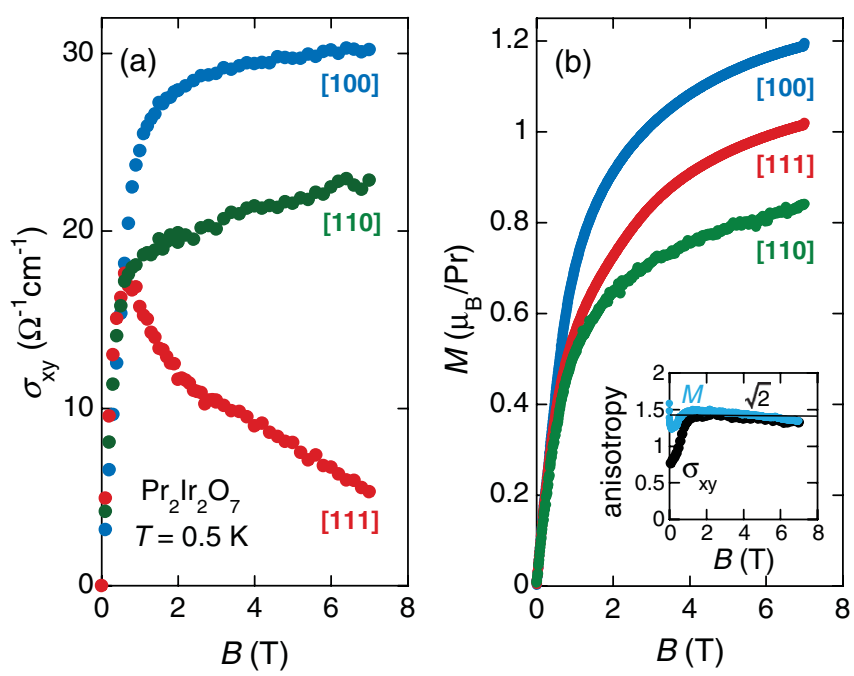

FIG. 3 (color online). Field dependence of (a) the Hall conductivity $\sigma_{x y}(B)$ and (b) the magnetization $M(B)$ at $T=0.5 \mathrm{~K}$ for different field directions. Inset: field dependence of the anisotropy of the Hall conductivity $\sigma_{x y}^{[100]} / \sigma_{x y}^{[110]}$ and the magnetization $M^{[100]} / M^{[110]}$ at $T=0.5 \mathrm{~K}$.

dences of the Hall conductivity $\sigma_{x y}(B)\left(\approx \rho_{x y}(B) / \rho_{x x}^{2}(B)\right)$ and $M(B)$ along the principal axes [100], [110], and [111] measured at $T=0.5 \mathrm{~K}\left(\ll \theta_{w}=1.7 \mathrm{~K}\right)$. Remarkably, $\sigma_{x y}(B)$ reaches a large value $\sim 25 \Omega^{-1} \mathrm{~cm}^{-1}$ under a relatively small field of about $1 \mathrm{~T}$, and eventually more than $30 \Omega^{-1} \mathrm{~cm}^{-1}$ at $7 \mathrm{~T}$. These are comparable with or larger than the values for metallic or semiconducting ferromagnets such as $(\mathrm{Ga}, \mathrm{Mn}) \mathrm{As}\left(14 \Omega^{-1} \mathrm{~cm}^{-1}\right)[5], \mathrm{Fe}_{1-x} \mathrm{Co}_{x} \mathrm{Si}$ $\left(40 \Omega^{-1} \mathrm{~cm}^{-1}\right)$ [6] and $\mathrm{Nd}_{2} \mathrm{Mo}_{2} \mathrm{O}_{7}\left(20 \Omega^{-1} \mathrm{~cm}^{-1}\right)$ [14]. Up to $B_{c} \sim 0.7 \mathrm{~T}$, both $\sigma_{x y}(B)$ and $M(B)$ is nearly isotropic and shows rapid and linear increase with field. This suggests that the spin-liquid-like state is stable below $B_{c}$ because the energy scale of $B_{c} \sim 0.7 \mathrm{~T}$ corresponds to that of $\theta_{w}=1.7 \mathrm{~K}$, the formation temperature of the spin-liquidlike state. However, beyond $B_{c}$, the field dependence becomes strongly anisotropic and is no longer proportional to $M$. For the [100] direction, for instance, $\sigma_{x y}$ becomes nearly saturated around $3 \mathrm{~T}$, while the corresponding $M$ is smoothly increasing with field. Furthermore, the [111] component of $\sigma_{x y}$ peaks around $B_{c}$, at which field no anomaly of $M$ was found. These anisotropic behavior and nonmonotonic $M$ dependence can be also clearly seen in the inset of Fig. 2.

In terms of the conventional mechanism due to the spinorbit coupling, it is highly difficult to understand the above nontrivial temperature and field dependences of the large Hall effect observed below $\theta_{w}=1.7 \mathrm{~K}$. Instead, it is natural to expect the spin-chirality contribution to the Hall effect because the $\operatorname{Pr}\langle 111\rangle$ Ising-like spins under fields may well have a sizeable spin chirality due to their noncoplanar spin texture and produce a fictitious magnetic field on the Ir sites through the Kondo coupling. This possibility has been discussed in $\mathrm{Nd}_{2} \mathrm{Mo}_{2} \mathrm{O}_{7}$, which also shows nonmonotonic $M$ dependence of $\sigma_{x y}$ [14].

In order to estimate the spin-chirality contribution to the Hall conductivity, we have to first clarify the spin structure under fields. While the spin configuration under zero field has not been clarified yet, those under the high field limit can be uniquely determined by the Zeeman energy scale, such as "2-in, 2-out" configuration for the [100] field direction, and "1-in, 3-out" (or "3-in, 1-out") configuration for the [111] field direction. For [110], however, half of the spins are perpendicular to the field, whose directions must be determined by the internal interactions. Thus, the "2-in, 2-out" state is most likely to be stabilized owing to the ferromagnetic nearest neighbor interactions [23].

The fictitious magnetic field $\boldsymbol{b}_{\text {Ir }}$ that penetrates a single Ir tetrahedron is defined as a vector sum:

$$
\boldsymbol{b}_{\text {Ir }}=\sum_{\langle i, j, k\rangle}\left\{\boldsymbol{I}_{i} \cdot\left(\boldsymbol{I}_{j} \times \boldsymbol{I}_{k}\right)\right\} \boldsymbol{n}_{i j k},
$$

where $\boldsymbol{I}$ is an internal field at an Ir site generated by $\operatorname{Pr}$ spins via the Kondo coupling, and $\boldsymbol{n}_{i j k}$ is a normal vector (with unit length) of a triangle formed by site $i, j$ and $k$ $[11,15]$. Figure 4 shows the configuration of the Pr spins (green arrows) for the "2-in, 2-out" and "1-in, 3-out" states and corresponding internal fields at the Ir sites (blue arrows). The Pauli paramagnetic Ir spins are polarized parallel to $\boldsymbol{I}$ at low $T$ if the Kondo effect due to the antiferromagnetic $f$ - $d$ exchange interaction $J_{f d}$ is dominant over the Zeeman energy effect. This is valid below about $B_{K} \sim 14 \mathrm{~T}$, which corresponds to the energy scale of (a) $\boldsymbol{B} / /[100]$

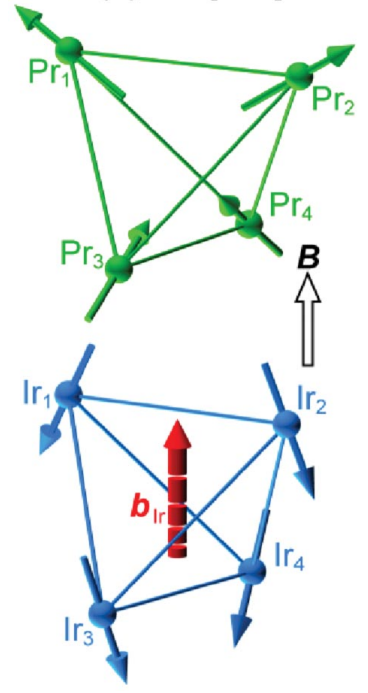

(b) $B / /[111]$

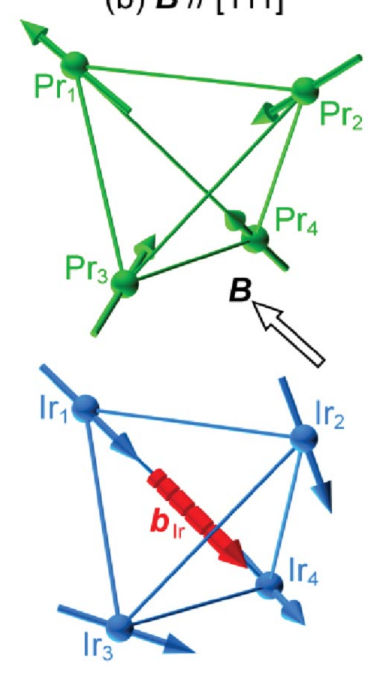

FIG. 4 (color online). Configurations of the Pr spins (green arrows) for the "2-in, 2-out" and "1-in, 3-out" states and the corresponding internal fields at the Ir sites (blue arrows) under the high field limit along the (a) [100] and (b) [111] directions. Open black and closed red arrows denote the directions of external and fictitious magnetic fields, respectively. 
$T_{K} \sim 20 \mathrm{~K}$. The direction of $\boldsymbol{I}$ depends on the configuration of the nearest neighbor Pr spins surrounding the Ir site. For instance, $\boldsymbol{I}_{1}$ at the $\operatorname{Ir}_{1}$ site is defined as $\boldsymbol{I}_{1}=$ $-\left(2 J_{f d} / \sqrt{3}\right)\left(S_{3}+S_{3}+S_{4}\right)$, where $\boldsymbol{S}_{i}$ is the $\operatorname{Pr}_{i}$ site spin with the size $|S|$, because the $\operatorname{Ir}_{1}$ site is located at a center of a Pr hexagon consisting of three pairs of equivalent sites to $\mathrm{Pr}_{2}, \mathrm{Pr}_{3}$, and $\mathrm{Pr}_{4}$. For the case of the "2-in, 2-out" and " 1 -in, 3-out" configurations, $\quad \boldsymbol{I}_{1}^{2 \downarrow, 2 \uparrow}=A(1,1,-3)$ and $\boldsymbol{I}_{1}^{1 \downarrow, 3 \uparrow}=A(-1,-1,-1)$, respectively, where $A \equiv$ $2 J_{f d}|S| / \sqrt{3}$. Then, $\boldsymbol{b}_{\text {Ir }}$ produced by the internal field of the Pr spins can be calculated for each case using Eq. (2), as $\boldsymbol{b}_{\mathrm{Ir}}^{2 \downarrow, 2 \uparrow}=48 A^{3}(1,0,0)$ and $\boldsymbol{b}_{\mathrm{Ir}}^{1,, 3 \uparrow}=24 A^{3}(-1,-1,-1)$. It is important to note that $\boldsymbol{b}_{\mathrm{Ir}}^{2 \downarrow, 2 \uparrow} \cdot \boldsymbol{B}$ is positive, while $\boldsymbol{b}_{\mathrm{Ir}}^{11,3 \uparrow} \cdot \boldsymbol{B}$ is negative as shown in Fig. 4.

Based on the spin-chirality mechanism, the Hall conductivity is proportional to the sum of $\boldsymbol{b}_{\mathrm{Ir}} \cdot \boldsymbol{B}$ over all the Ir tetrahedra. As we discussed above, with fields along [100] and [110], the "2-in, 2-out" state becomes stabilized and generates the fictitious magnetic field $\boldsymbol{b}_{\mathrm{Ir}}^{2 \mathrm{l}, 2 \uparrow}$ along the $\langle 100\rangle$ direction. This should be the reason why strongly enhanced $\sigma_{x y}$ is observed for both [100] and [110] directions. On the other hand, along the [111] direction, the number of the tetrahedra with the "1-in, 3-out" state should increase as a function of field. Since $\boldsymbol{b}_{\mathrm{Ir}}^{1 \downarrow, 3 \uparrow} \cdot \boldsymbol{B}$ has the opposite sign to $\boldsymbol{b}_{\mathrm{Ir}}^{2 \downarrow, 2 \uparrow} \cdot \boldsymbol{B}, \sigma_{x y}^{[111]}$ is expected to decrease and become negative under high fields. Indeed, $\sigma_{x y}^{[111]}$ peaks at around $B_{c}$ and starts decreasing with fields, suggesting that it is $B_{c}$ where the crossover starts from the spin-liquid-like regime to the "1-in, 3-out" state. On the other hand, $\sigma_{x y}^{[111]}$ remains positive up to $7 \mathrm{~T}$, probably because of the additional contribution based on the conventional mechanism.

Furthermore, based on the spin-chirality mechanism, the anisotropy of $\sigma_{x y}^{[100]} / \sigma_{x y}^{[110]}$ should be given simply by the inverse cosine of the angle between [100] and [110] because for both cases, the fictitious magnetic field should be along the $\langle 100\rangle$ direction. This is indeed the case, as shown in the inset of Fig. 3(b), and $\sigma_{x y}^{[100]} / \sigma_{x y}^{[110]}$ stays almost close the expected ratio of $\sqrt{2}$ above 1 T. However, this does not necessarily mean that the conventional contribution based on Eq. (1) is negligible. Given that the magnetization anisotropy $M^{[100]} / M^{[110]}$ is nearly $\sqrt{2}$ owing to the $\langle 111\rangle$ Ising anisotropy of the Pr moments [inset of Fig. 3(b)], the ratio of the expected conventional contributions $4 \pi R_{S} M$ for these two field directions would also be close to $\sqrt{2}$, if $R_{s}$ is nearly isotropic in these two field directions at low $T$. To quantify such contributions, we are planning to extend our measurements of $\sigma_{x y}$ to high magnetic fields. Once the field becomes much larger than $B_{K} \sim 14 \mathrm{~T}$, the Ir conduction electron spins should be fully polarized to have zero chirality, and thus $\sigma_{x y}$ is expected to approach certain values originating from the conventional mechanism for all directions.
In fact, in $\mathrm{Nd}_{2} \mathrm{Mo}_{2} \mathrm{O}_{7}$, all the direction components of $\sigma_{x y}$ decrease above several teslas corresponding to the energy scale of $J_{f d}$ and saturate at finite values under high fields [15]. However, in our case of $\operatorname{Pr}_{2} \mathrm{Ir}_{2} \mathrm{O}_{7}, J_{f d}$ should be larger than $B_{K} \sim 14 \mathrm{~T}$, and thus the fictitious magnetic field made of the Pr spin chirality may well be strong enough to induce the large Hall effect, as observed up to $7 \mathrm{~T}$. Furthermore, the maximum of the Hall conductivity $30 \Omega^{-1} \mathrm{~cm}^{-1}$ is larger than that of the ferromagnet $\mathrm{Nd}_{2} \mathrm{Mo}_{2} \mathrm{O}_{7}\left(20 \Omega^{-1} \mathrm{~cm}^{-1}\right)$. This fact is quite remarkable because $\operatorname{Pr}_{2} \mathrm{Ir}_{2} \mathrm{O}_{7}$ is paramagnetic without any spontaneous magnetization. This underlines the Pauli paramagnetic state of the Ir conduction electrons, which allows the Ir electron spins to subtend the solid angle as wide as that determined by the $\operatorname{Pr}$ spin chirality. The large $\sigma_{x y}$ observed in the spin-liquid-like paramagnetic state of $\operatorname{Pr}_{2} \mathrm{Ir}_{2} \mathrm{O}_{7}$ sheds new light on the approach to enhance the Hall effect in magnetic conductors.

We thank H. Kawamura, C. Lacroix, N. Nagaosa, and G. Tatara for discussions. This work is supported in part by Grants-in-Aid for Scientific Research from JSPS and for the 21st Century COE "Center for Diversity and Universality in Physics" from MEXT of Japan, and by the Nippon Sheet Glass Foundation.

[1] C. M. Hurd, The Hall Effect in Metals and Alloys (Plenum Press, NewYork, 1972).

[2] R. Karplus and J.M. Luttinger, Phys. Rev. 95, 1154 (1954).

[3] J. Smit, Physica (Amsterdam) 21, 877 (1955).

[4] L. Berger, Phys. Rev. B 2, 4559 (1970).

[5] F. Matsukura et al., Phys. Rev. B 57, R2037 (1998).

[6] N. Manyala et al., Nat. Mater. 3, 255 (2004).

[7] P. Matl et al., Phys. Rev. B 57, 10248 (1998).

[8] J. Ye et al., Phys. Rev. Lett. 83, 3737 (1999).

[9] K. Ohgushi, S. Murakami, and N. Nagaosa, Phys. Rev. B 62, R6065 (2000).

[10] G. Tatara and H. Kawamura, J. Phys. Soc. Jpn. 71, 2613 (2002).

[11] S. Onoda and N. Nagaosa, Phys. Rev. Lett. 90, 196602 (2003).

[12] M. Taillefumier, Phys. Rev. B 74, 085105 (2006).

[13] S. Yoshii et al., J. Phys. Soc. Jpn. 69, 3777 (2000).

[14] Y. Taguchi et al., Science 291, 2573 (2001).

[15] Y. Taguchi et al., Phys. Rev. Lett. 90, 257202 (2003).

[16] Y. Yasui et al., J. Phys. Soc. Jpn. 75, 084711 (2006).

[17] D. Yanagishima and Y. Maeno, J. Phys. Soc. Jpn. 70, 2880 (2001).

[18] S. Nakatsuji et al., Phys. Rev. Lett. 96, 087204 (2006).

[19] Y. Machida et al., J. Phys. Chem. Solids 66, 1435 (2005).

[20] J. M. Millican et al., Mater. Res. Bull. (to be published).

[21] T. Sakakibara et al., Jpn. J. Appl. Phys. 33, 5067 (1994).

[22] S. Onoda, N. Sugimoto, and N. Nagaosa, Phys. Rev. Lett. 97, 126602 (2006).

[23] M. J. Harris et al., Phys. Rev. Lett. 81, 4496 (1998). 